Article

\title{
Vibro-Impact Energy Analysis of a Geared System with Piecewise-Type Nonlinearities Using Various Parameter Values
}

\section{Jong-Yun Yoon ${ }^{1}$ and Byeongil Kim ${ }^{2, *}$}

1 Department of Mechanical and Automotive Engineering, Kongju National University, Cheonan-si, Chungcheongnam-do 314-701, Korea; E-Mail: yoon3932@kongju.ac.kr

2 School of Mechanical Engineering, Yeungnam University, Gyeongsan-si, Gyeongsangbuk-do 712-749, Korea

* Author to whom correspondence should be addressed; E-Mail: bikim@yu.ac.kr; Tel.: +82-53-810-2447; Fax: +82-53-810-4627.

Academic Editor: Frede Blaabjerg

Received: 6 July 2015 / Accepted: 14 August 2015 / Published: 21 August 2015

\begin{abstract}
Torsional systems with gear pairs such as the gearbox of wind turbines or vehicle driveline systems inherently show impact phenomena due to clearance-type nonlinearities when the system experiences sinusoidal excitation. This research investigates the vibro-impact energy of unloaded gears in geared systems using the harmonic balance method (HBM) in both the frequency and time domains. To achieve accurate simulations, nonlinear models with piecewise and clearance-type nonlinearities and drag torques are defined and implemented in the HBM. Next, the nonlinear frequency responses are examined by focusing on the resonance areas where the impact phenomena occur, along with variations in key parameters such as clutch stiffness, drag torque, and inertias of the flywheel and the unloaded gear. Finally, the effects of the parameters on the vibro-impacts at a specific excitation frequency are explained using bifurcation diagrams. The results are correlated with prior research by defining the gear rattle criteria with key parameters. This article suggests a method to simulate the impact phenomena in torsional systems using the HBM and successfully assesses vibro-impact energy using bifurcation diagrams.
\end{abstract}

Keywords: vibro-impact energy; wind turbines; multi-staged clutch damper; harmonic balance method; drag torque; firing frequency; gear backlash; clearance 


\section{Introduction}

A practical gearbox system for wind turbines or vehicle manual transmissions under excitation conditions shows severe vibro-impacts due to clearance-type nonlinearities [1-9]. In general, clearance-type nonlinearties mean the nonlinear characteristics caused by the clearance such as a distance between two sub-systems. For example, a gear backlash is a representative clearance located between two gear teeth where repetitive separation and contact motions are caused by various engine input conditions, which produce severe vibro-impacts due to the alternating engine input torque flow. Regarding these vibro-impacts, various methods have been suggested to understand the impact phenomena caused by clearances such as gear backlash. For example, Padmanabhan and Singh [1] employed the harmonic balance method (HBM) to simulate neutral gear rattle in an automotive transmission. Kim et al. [3] examined impact damping with clearance-type nonlinearities with focus on gear backlash. Yoon and Lee [8] used two smoothening factors to investigate practical nonlinear dynamic behaviors due to piecewise-type nonlinearities. Yoon and Yoon [9] employed the HBM to investigate the dynamic characteristics of a single-degree-of-freedom (DOF) torsional system accompanied by a multi-stage clutch damper model. Other studies have examined various vibration problems using the HBM [10-22]. For instance, nonlinear problems using a Duffing oscillator or cubic stiffness have been settled by utilizing the nonlinear output frequency response functions (NOFRFs) and incremental harmonic balance (IHB) method [10,11]. Also, chaotic behaviors of physical systems have been examined $[12,13]$. Additionally, the response of a system with a hysteretic restoring force has been studied by applying two degree of freedom chain systems with sinusoidal inputs [14]. Incremental or multi-component HBMs have been employed as well to investigate the nonlinear system responses [15-18]. The time domain responses for the $i^{\text {th }}$ sub-system have been extended based upon the Galerkin scheme [19-22]. Although many studies have investigated clearance-type nonlinearities, difficulties still remain in implementing these methods on practical systems.

As an example of geared systems, a manual transmission driveline is introduced for this research and the dynamic behaviors of such systems can be generally observed in other geared systems such as wind turbines. As the theoretical background of this study contains the concepts of gear contact/separation as well as transmission errors describing the relationship of gear mesh forces $v s$. relative displacement of the gear pair, the scope of this research can be adapted to macro scale geared system such as wind turbine system drivelines. Figure 1 shows a reduced-order model of a manual transmission driveline, which is an example of a physical system, with pertinent nonlinear elements [7,8]. It is a 5-speed manual transmission model with parallel type gears. The transmission is assumed to be under the third gear engaged and the fifth gear unloaded status. The order of the original system has been reduced so that most of the speed gears are unloaded and rotating except for one engaged through the synchronizer and gear-shifting mechanism. The key components such as the gear pairs, the clutch hub, the flywheel, the input shaft, the axles and the tire-vehicle system are characterized by the lumped torsional inertia $I$ or stiffness $k$. The fixed gears and the synchronizer assembly are considered as the input and output shafts. The reduced model with six DOF is the minimum level of system that can accommodate all the key parameters, such as the multi-stage clutch dampers, drag torques, and gear backlash on both engaged and unloaded gear pairs. These nonlinearities are illustrated in the schematic diagram shown in Figure 1. Definitions of the key parameters are listed in Table 1. 


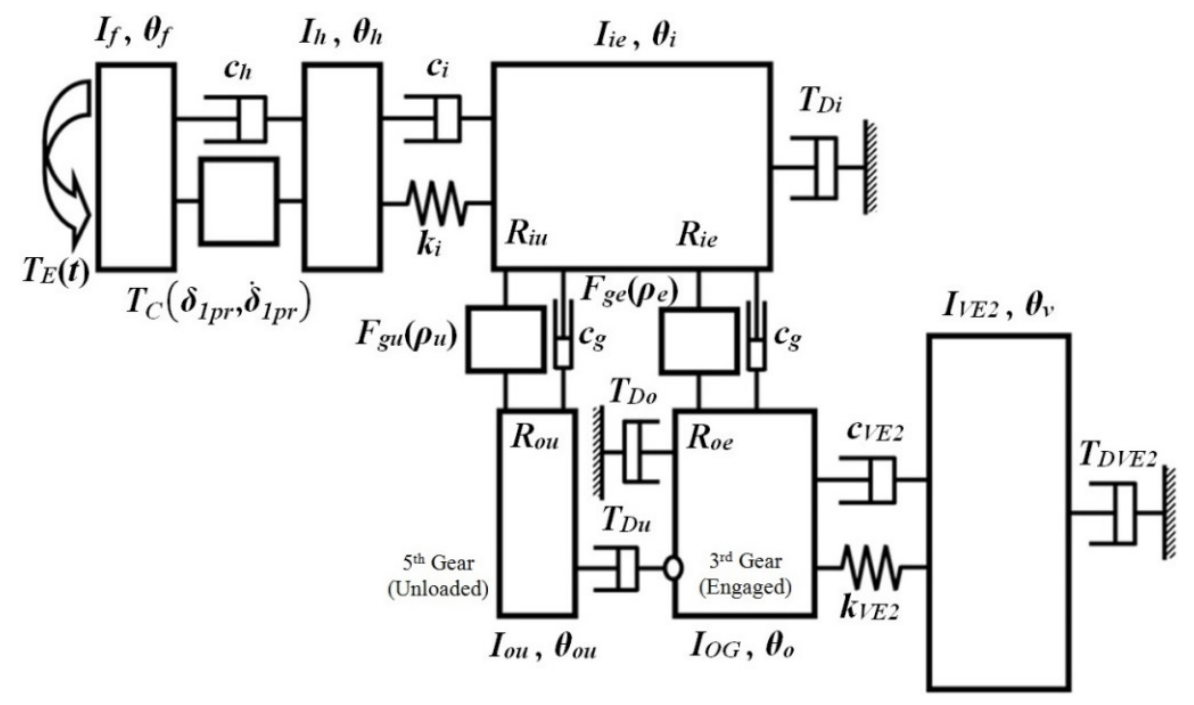

Figure 1. A nonlinear torsional system model with six degrees of freedom with multiple nonlinearites.

Table 1. Definitions of key parameters.

\begin{tabular}{cccc}
\hline Key parameters & Definition & Key parameters & Definition \\
\hline$F_{g u}$ & Gear mesh force of unloaded gear & $\theta_{h}$ & Absolute motion of clutch hub \\
$F_{g e}$ & Gear mesh force of engaged gear & $\theta_{i}$ & Absolute motion of input shaft \\
$T_{E}$ & Input (engine) torque & $\theta_{o u}$ & Absolute motion of unloaded gear \\
$T_{C}$ & Overall clutch torque & $\theta_{o}$ & Absolute motion of output shaft \\
$T_{D i}$ & Drag torque on input shaft & $\theta_{v}$ & Absolute motion of vehicle \\
$T_{D o}$ & Drag torque on output shaft & $c_{h}$ & Damping of clutch hub \\
$T_{D u}$ & Drag torque on unloaded gear & $c_{i}$ & Damping of input shaft \\
$T_{D V E 2}$ & Drag torque on vehicle & $c_{g}$ & Damping of gear mesh \\
$I_{f}$ & Inertia of flywheel & $c_{V E 2}$ & Damping of vehicle \\
$I_{h}$ & Inertia of clutch hub & $k_{i}$ & Stiffness of input shaft \\
$I_{i e}$ & Inertia of input shaft & $k_{V E 2}$ & Stiffness of vehicle \\
$I_{o u}$ & Inertia of unloaded gear & $R_{i u}$ & Radius of unloaded gear on input shaft \\
$I_{O G}$ & Inertia of output shaft & $R_{i e}$ & Radius of engaged gear on input shaft \\
$I_{V E 2}$ & Inertia of vehicle & $R_{o u}$ & Radius of unloaded gear on output shaft \\
$\theta_{f}$ & Absolute motion of flywheel & $R_{o e}$ & Radius of engaged gear on output shaft \\
\hline
\end{tabular}

Figure 2 illustrates the piecewise-type nonlinearity between the flywheel and clutch hub and clearance-type nonlinearity between engaged and unloaded gear pairs [7-9]. In this figure, clutch torque profiles are expressed including hysteresis levels and transition angles. A nonlinear function $T_{C}$ has been depicted by the relationship of clutch torque $v s$. relative displacement $\delta_{1 P r}$. This profile is measured under the static load conditions when the torque levels of multi-staged linear torsional spring are determined by both $\delta_{1 P r}$ and $\dot{\delta}_{1 P r}$, which is defined as the relative velocity between clutch and flywheel. $T_{C}, \delta_{1 p r}$, and $k_{C n}$ indicate overall clutch torque, relative displacement between the flywheel and the clutch hub, and torsional stiffness of $n^{\text {th }}$ stage clutch damper, respectively. Figure $2 \mathrm{~b}$ indicates the expected gear mesh force with the relationship of the relative displacement of the gear pair $\delta v s$. the gear mesh force $F$. Here, $k_{g}$ and $b$ indicate the gear mesh stiffness and the gear backlash, respectively. Observing this figure, 
the dynamic conditions of the forces are abruptly changed at $b / 2$ or $-b / 2$. To simulate strong nonlinearities at multiple points in the torsional system, the HBM will be used by extending the previously proposed basic formulations [9,22]. In addition, this study examines the nonlinear behaviors of an impact pair from the geared systems, in the frequency domain, by extending prior impact pair analysis [23].

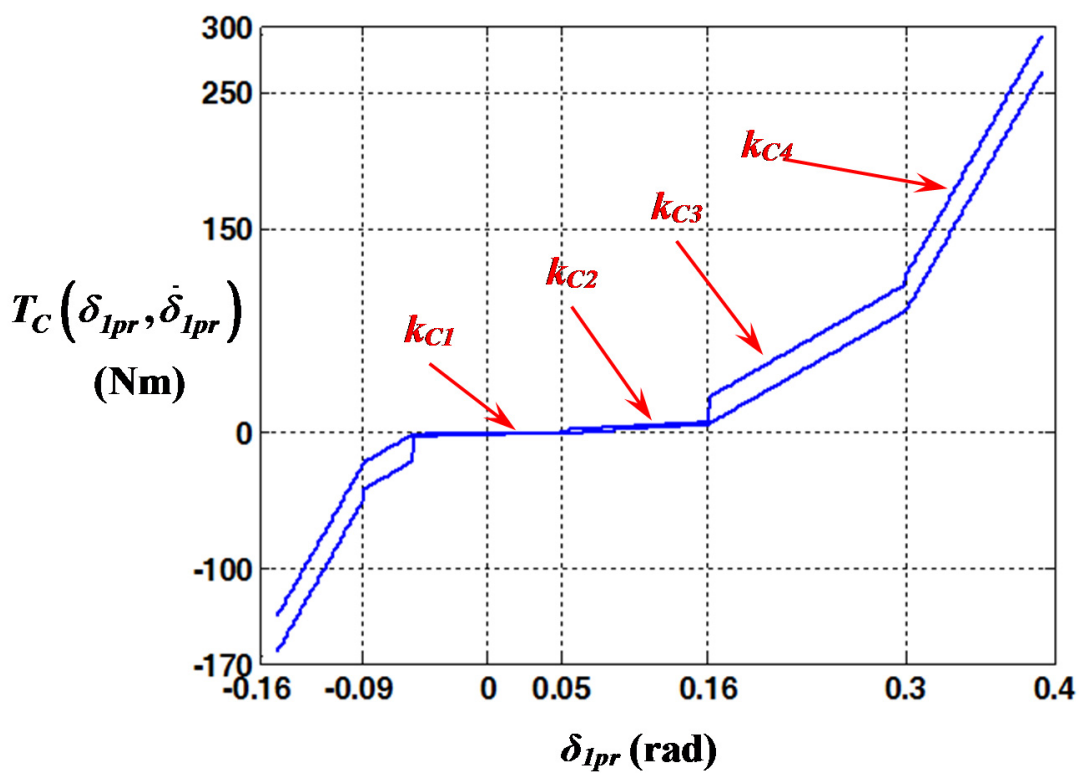

(a)

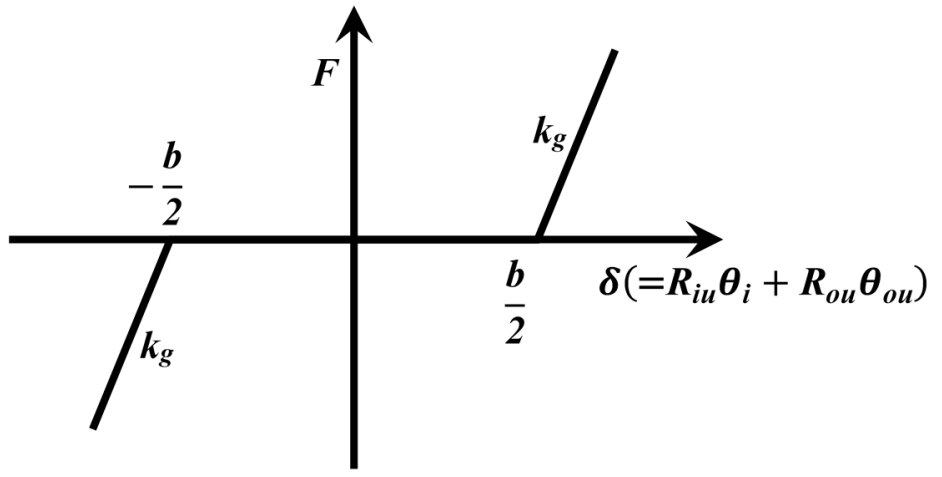

(b)

Figure 2. Nonlinear characteristics of multi-stage clutch dampers and gear backlash: (a) Nonlinear function $T_{C}\left(\delta_{1 p r}, \dot{\delta}_{1 p r}\right)$ for multi-stage clutch dampers; (b) Gear mesh force.

The specific objectives of this study are as follows: (1) the dynamic behaviors of impact pairs in a geared system were investigated by implementing piecewise-type nonlinearities at multiple points; (2) the effects of key parameters on the vibro-impacts were examined using the HBM to improve the vibratory conditions; and (3) the impact phenomena were numerically mapped in the bifurcation diagrams for the key parameters, which provides insight into the gear rattle criteria. Overall, within the scope described above, this research could help readers understand the basic concepts of the geared systems so that they can develop specific simulation methods of highly nonlinear systems such as wind turbines. 


\section{Problem Formulation with 6-DOF Nonlinear System Model}

Based on the nonlinear system shown in Figure 1, the basic equations are derived as follows [7-9]. A reduced 6-DOF torsional system model is considered in this research, since a larger dimension may cause problems for nonlinear analysis. It includes nonlinear terms describing the clutch torque $T_{C}$, unloaded gear force $F_{g u}$, and engaged gear force $F_{g e}$. The governing equations are derived from the moment equilibrium of each segment and placed in the matrix form. Assuming that drag torques are constant, the governing equations are given as:

$$
\begin{gathered}
I_{f} \ddot{\theta}_{f}(t)+c_{h}\left\{\dot{\theta}_{f}(t)-\dot{\theta}_{i}(t)\right\}+T_{C}\left(\delta_{1 p r}, \dot{\delta}_{1 p r}\right)=T_{E}(t) \\
I_{h} \ddot{\theta}_{h}(t)-c_{h} \dot{\theta}_{f}(t)+\left(\mathrm{c}_{h}+c_{i}\right) \dot{\theta}_{h}(t)-c_{i} \dot{\theta}_{i}(t)+k_{i} \theta_{h}(t)-k_{i} \theta_{i}(t)=T_{C}\left(\delta_{1 p r}, \dot{\delta}_{1 p r}\right) \\
I_{i e} \ddot{\theta}_{i}(t)-c_{i} \dot{\theta}_{h}(t)+\left(c_{i}+c_{g e} R_{i e}^{2}+c_{g u} R_{i u}^{2}\right) \dot{\theta}_{i}(t)+c_{g u} R_{i u} R_{o u} \dot{\theta}_{o u}(t)+c_{g e} R_{i e} R_{o e} \dot{\theta}_{o}(t) \\
-k_{i} \theta_{h}(t)+k_{i} \theta_{i}(t)=-R_{i u} F_{g u}\left(\rho_{u}\right)-R_{i e} F_{g e}\left(\rho_{e}\right)-\mathrm{T}_{D i} \\
I_{o u} \ddot{\theta}_{o u}(t)+\mathrm{c}_{g u} R_{i u} R_{o u} \dot{\theta}_{i}(t)+c_{g u} R_{i u}^{2} \dot{\theta}_{o u}(t)=-R_{o u} F_{g u}\left(\rho_{u}\right)+T_{D u} \\
I_{O G} \ddot{\theta}_{o}(t)+c_{g e} R_{i e} R_{o e} \dot{\theta}_{i}(t)+\left(c_{g e} R_{o e}^{2}+c_{V E 2}\right) \dot{\theta}_{o}(t)-\mathrm{c}_{V E 2} \dot{\theta}_{V E 2}(t) \\
+k_{V E 2} \theta_{o}(t)-k_{V E 2} \theta_{V E 2}(t)=-R_{o e} F_{g e}\left(\rho_{e}\right)+T_{D o} \\
I_{V E 2} \ddot{\theta}_{v}(t)-c_{V E 2} \dot{\theta}_{o}(t)+c_{V E 2} \dot{\theta}_{v}(t)-k_{V E 2} \theta_{o}(t)+k_{V E 2} \theta_{v}(t)=T_{D V E 2}
\end{gathered}
$$

Here, $\theta_{k}(t)(k=f, h, i, o u, o, v)$ is the absolute motions of sub-systems such as the flywheel, clutch hub, input shaft, unloaded gear, output shaft, and vehicle. Equations (1a)-(1f) can be expressed in the matrix formulation by defining a state vector $\underline{\boldsymbol{\theta}}(t)=\left[\begin{array}{llllll}\theta_{f} & \theta_{h} & \theta_{i} & \theta_{o u} & \theta_{o} & \theta_{v}\end{array}\right]^{T}$ as follows.

$$
\begin{aligned}
& \underline{\underline{\mathbf{M}}} \ddot{\underline{\boldsymbol{\theta}}}(t)+\underline{\underline{\mathbf{C}}} \underline{\dot{\boldsymbol{G}}}(t)+\underline{\underline{\mathbf{K}}}(t)+\underline{\mathbf{f}}(\underline{\boldsymbol{\theta}}, \underline{\dot{\boldsymbol{\theta}}})=\underline{\mathbf{T}_{\mathbf{E}}}(t) \\
& \underline{\underline{\mathbf{M}}}=\operatorname{diag}\left[I_{f}, I_{h}, I_{i e}, I_{o u}, I_{O G}, I_{V E 2}\right] \\
& \underline{\mathbf{C}}=\left[\begin{array}{cccccc}
c_{h} & -c_{h} & 0 & 0 & 0 & 0 \\
-c_{h} & c_{h}+c_{i} & -c_{i} & 0 & 0 & 0 \\
0 & -c_{i} & c_{i}+c_{g}\left(R_{i e}^{2}+R_{i u}^{2}\right) & c_{g} R_{i u} R_{o u} & c_{g} R_{i e} R_{o e} & 0 \\
0 & 0 & c_{g} R_{i u} R_{o u} & c_{g} R_{o u}^{2} & 0 & 0 \\
0 & 0 & c_{g} R_{i e} R_{o e} & 0 & c_{g} R_{o e}^{2}+c_{V E 2} & -c_{V E 2} \\
0 & 0 & 0 & 0 & -c_{V E 2} & c_{V E 2}
\end{array}\right] \\
& \underline{\underline{\mathbf{K}}}=\left[\begin{array}{cccccc}
0 & 0 & 0 & 0 & 0 & 0 \\
0 & k_{i} & -k_{i} & 0 & 0 & 0 \\
0 & -k_{i} & k_{i} & 0 & 0 & 0 \\
0 & 0 & 0 & 0 & 0 & 0 \\
0 & 0 & 0 & 0 & k_{V E 2} & -k_{V E 2} \\
0 & 0 & 0 & 0 & -k_{V E 2} & k_{V E 2}
\end{array}\right]
\end{aligned}
$$




$$
\underline{\mathbf{f}_{\mathbf{n}}}(\underline{\boldsymbol{\theta}}, \underline{\boldsymbol{\theta}})=\left[\begin{array}{c}
T_{C}\left(\delta_{1 p r}, \dot{\delta}_{1 p r}\right) \\
-T_{C}\left(\delta_{1 p r}, \dot{\delta}_{1 p r}\right) \\
R_{i u} F_{g u}\left(\rho_{u}\right)+R_{i e} F_{g e}\left(\rho_{e}\right)+T_{D i} \\
R_{o u} F_{g u}\left(\rho_{u}\right)-T_{D u} \\
R_{o e} F_{g e}\left(\rho_{e}\right)-T_{D o} \\
-T_{D V E 2}
\end{array}\right], \underline{\mathbf{T}_{\mathbf{E}}}(t)=\left[\begin{array}{c}
T_{E}(t) \\
0 \\
0 \\
0 \\
0 \\
0
\end{array}\right]
$$

Here, $\mathbf{M}, \mathbf{C}$, and $\mathbf{K}$ are inertia, damping, and stiffness matrices, respectively, and $\mathbf{f}_{\mathbf{n}}(\underline{\boldsymbol{\theta}}, \underline{\dot{\boldsymbol{\theta}}})$ and $\mathbf{T}_{\mathbf{E}}(t)$ are a nonlinear function and input torque vectors respectively. The other parameter designations and the values used in the simulation are summarized in Tables 2 and 3 [7-9,22]. The employed nonlinear models can be derived as follows [7,8]. The nonlinear function describing the overall clutch torque $T_{C}$ is defined as the summation of the preload torque $T_{S P r}$, stiffness torque $T_{S}$, and hysteresis torque $T_{H}$ :

$$
T_{C}\left(\delta_{1 p r}, \dot{\delta}_{1 p r}\right)=T_{S P r}\left(\delta_{1 p r}\right)+T_{S}\left(\delta_{1 p r}\right)+T_{H}\left(\delta_{1 p r}, \dot{\delta}_{1 p r}\right)
$$

The torque caused by the preload $T_{S P r}$ is determined by the function of relative displacement $\delta_{1 p r}$ :

$$
T_{S P r}\left(\delta_{1 p r}\right)=\frac{1}{2} T_{P r 1}\left[\tanh \left(\sigma_{C} \delta_{1 p r}\right)+1\right]+\frac{1}{2} T_{P r 2}\left[-\tanh \left(\sigma_{C} \delta_{1 p r}\right)+1\right], \delta_{1 p r}=\delta_{1}-\phi_{P r}
$$

Here, $T_{P r 1}$ and $T_{P r 2}$ indicate the positive and negative preload, $\sigma_{C}$ is a smoothening factor, and $\varphi_{\operatorname{Pr}}$ is the phase at the preload when $\delta_{1}$ is zero, respectively. The clutch torque resulted by the stiffness $T_{S}\left(\delta_{1}\right)$ is defined as follows:

$$
\begin{gathered}
T_{S}\left(\delta_{1 p r}\right)=k_{C 1} \delta_{1 p r}+\frac{1}{2} \sum_{i=2}^{N}\left(k_{C(i)}-k_{C(i-1)}\right)\left(D_{s p(i-1)}-D_{s n(i-1)}\right) \\
D_{s p(i)}=\left(\delta_{1 p r}-\phi_{p(i)}\right)\left[\tanh \left\{\sigma_{C}\left(\delta_{1 p r}-\phi_{p(i)}\right)\right\}+1\right] \\
D_{s n(i)}=\left(\delta_{1 p r}+\phi_{n(i)}\right)\left[\tanh \left\{\sigma_{C}\left(\delta_{1 p r}+\phi_{n(i)}\right)\right\}-1\right]
\end{gathered}
$$

In these equations, $k c(i)$ is the clutch stiffness, $D_{s p}(i)$ and $D_{s n}(i)$ are the positive and negative sides of the clutch displacements induced by stiffness at the $i^{\text {th }}$ stage, and $\varphi_{p}(i)$ and $-\varphi_{n}(i)$ are the $i^{\text {th }}$ transition angles of the positive and negative sides. The torque due to hysteresis $T_{H}$ for the multi-staged clutch under asymmetric transition angles is determined as follows:

$$
\begin{gathered}
T_{H}\left(\delta_{1 p r}, \dot{\delta}_{1 p r}\right)=\frac{H_{(N)}}{2} \tanh \left(\sigma_{H} \dot{\delta}_{1 p r}\right)+\sum_{i=2}^{N}\left(\frac{H_{(i)}}{4}-\frac{H_{(i-1)}}{4}\right)\left[D_{H p(i-1)}+D_{H n(i-1)}\right] \\
D_{H p(i)}=\tanh \left\{\sigma_{C}\left(\delta_{1 p r}-\phi_{p(i)}\right)\right\}\left[1+\tanh \left(\sigma_{H} \dot{\delta}_{1 p r}\right)\right] \\
D_{H n(i)}=\tanh \left\{\sigma_{C}\left(\delta_{1 p r}+\phi_{n(i)}\right)\right\}\left[1-\tanh \left(\sigma_{H} \dot{\delta}_{1 p r}\right)\right]
\end{gathered}
$$

where $H(i)$ indicates the $i^{\text {th }}$ stage of hysteresis and $D_{H p}(i)$ and $D_{H n}(i)$ stand for the positive and negative sides of relative motions resulted by hysteresis at the $i^{\text {th }}$ stage, respectively. 
Table 2. Employed values of the torsional system parameters $[7,8]$.

\begin{tabular}{|c|c|c|c|c|c|}
\hline Inertia & $\begin{array}{c}\text { Value } \\
\left(\mathrm{kg} \cdot \mathrm{m}^{2}\right)\end{array}$ & Stiffness & $\begin{array}{c}\text { Value } \\
\left(\mathbf{N m} \cdot \mathbf{r a d}^{-1}\right) \\
\end{array}$ & Radius & $\begin{array}{l}\text { Value } \\
(\mathrm{mm})\end{array}$ \\
\hline $\begin{array}{c}I_{f} \\
\text { (Flywheel) }\end{array}$ & $1.38 \times 10^{-1}$ & $\begin{array}{c}k_{C} \\
\text { (Clutch for the linear model) }\end{array}$ & 1838.0 & $\begin{array}{c}R_{i e} \\
\text { (Engaged gear } \\
\text { on the input shaft) }\end{array}$ & 35.5 \\
\hline $\begin{array}{c}I_{h} \\
\text { (Clutch hub) }\end{array}$ & $5.76 \times 10^{-3}$ & $\begin{array}{c}k_{i} \\
\text { (Input shaft) }\end{array}$ & 10000 & $\begin{array}{c}R_{o e} \\
\text { (Engaged gear } \\
\text { on the output shaft) }\end{array}$ & 46.0 \\
\hline $\begin{array}{c}I_{i e} \\
\text { (Input shaft) }\end{array}$ & $4.53 \times 10^{-3}$ & $\begin{array}{c}k_{V E 2} \\
\text { (Drive shaft) }\end{array}$ & $6.63 \times 10^{2}$ & $\begin{array}{c}R_{i u} \\
\text { (Unloaded gear } \\
\text { on the input shaft) }\end{array}$ & 45.9 \\
\hline $\begin{array}{c}I_{O G} \\
\text { (Output shaft) }\end{array}$ & $7.80 \times 10^{-3}$ & $\begin{array}{c}k_{g} \\
\text { (Gear mesh) }\end{array}$ & $2.7 \times 10^{8}\left(\mathrm{Nm}^{-1}\right)$ & $\begin{array}{c}R_{o u} \\
\text { (Unloaded gear } \\
\text { on the output shaft) }\end{array}$ & 35.6 \\
\hline $\begin{array}{c}I_{o u} \\
\text { (Unloaded gear) }\end{array}$ & $5.23 \times 10^{-4}$ & & & & \\
\hline $\begin{array}{c}I_{V E 2} \\
\text { (Vehicle) } \\
\end{array}$ & 3.27 & & & & \\
\hline
\end{tabular}

The nonlinear function for the gear mesh force $F_{g}$ is defined as the following equation.

$$
\begin{gathered}
F_{g k}\left(\rho_{k}\right)=k_{g} \rho_{k}+k_{g} \frac{\left(\rho_{k}-\frac{b}{2}\right) \tanh \left\{\sigma_{g}\left(\rho_{k}-\frac{b}{2}\right)\right\}-\left(\rho_{k}+\frac{b}{2}\right) \tanh \left\{\sigma_{g}\left(\rho_{k}+\frac{b}{2}\right)\right\}}{2} \\
\rho_{k}=R_{i k} \theta_{i}+R_{o k} \theta_{o}(k=e \text { or } u)
\end{gathered}
$$

Here, $b$ is defined as the gear backlash and the subscripts $e$ and $u$ are "engaged' and 'unloaded". $\sigma_{g}$ is the smoothening factor in the calculation of the gear mesh force.

In Equations (3)-(7b), the nonlinear function for the overall clutch torque $T_{C}$ is the summation of the pre-load torque $T_{S P r}$, stiffness torque $T_{S}$, and hysteresis torque $T_{H}$. All of the clutch torques are expressed as a function of relative displacement $\delta_{l p r}$ given by Equation (4b), where $\phi_{P r}$ is the phase at the pre-load when the relative motion $\delta_{1}$ between the flywheel and clutch hub is zero [7-9]. $T_{\operatorname{Pr} 1}$ and $T_{\operatorname{Pr} 2}$ are the positive and negative pre-loads, and $\sigma_{C}, \sigma_{H}$, and $\sigma_{G}$ are smoothening factors for the clutch stiffness, hysteresis, and gear backlash, respectively. Values of $1 \times 10^{2}, 0.1$, and $1 \times 10^{10}$ are employed for $\sigma_{C}$, $\sigma_{H}$, and $\sigma_{G}$ to calculate the clutch torques induced by the stiffness, hysteresis, and gear mesh forces, respectively [7-9]. These smoothening factors are used for numerical convergence, especially for the stiff problem, and they are determined to minimize the difference between the physical discontinuities and smoothened simulation functions [7-9]. 
Table 3. Property values of the real-life multi-stage clutch damper $[7,8]$.

\begin{tabular}{ccc}
\hline Property & Stage & Value \\
\hline Torsional stiffness, $k_{C i}$ & 1 & 10.1 \\
(linearized in a piecewise manner) & 2 & 61.8 \\
$(\mathrm{Nm} / \mathrm{rad})$ & 3 & 595.8 \\
& 4 & 1838.0 \\
\hline & 1 & 0.98 \\
Hysteresis, $H_{i}(\mathrm{Nm})$ & 2 & 1.96 \\
& 3 & 19.6 \\
& 4 & 26.5 \\
\hline Transition angle at positive side & 1 & 0.05 \\
$\left(\delta_{i}>0\right),(\mathrm{rad})$ & 2 & 0.16 \\
& 3 & 0.30 \\
Transition angle at negative side & 4 & 0.39 \\
$\left(\delta_{i}<0\right),(\mathrm{rad})$ & 1 & -0.04 \\
& 2 & -0.05 \\
& 3 & -0.09 \\
& 4 & -0.15 \\
\hline
\end{tabular}

$k_{C(i)}$ is the clutch stiffness, $D_{s p(i)}$ and $D_{s n(i)}$ are the switching functions at positive and negative sides of clutch relative velocities at the $i^{\text {th }}$ stage [9], and $\phi_{p(i)}$ and $-\phi_{n(i)}$ are the $i^{\text {th }}$ transition angles of the positive and negative sides, respectively. $H_{(i)}$ is the $i^{\text {th }}$ stage of hysteresis, and $D_{H p(i)}$ and $D_{H n(i)}$ are the positive and negative sides of the relative motions induced by hysteresis at the $i^{\text {th }}$ stage, respectively. The nonlinear function for the gear mesh force $F_{g}$ is expressed by the gear backlash $b$, and subscripts $e$ and $u$ indicate engaged and unloaded states. The transitional relative displacements between gear pairs are denominated by $\rho_{e}$ and $\rho_{u}$. The properties employed for the multi-stage clutch damper are summarized in Table 3. A value of $0.1 \mathrm{~mm}$ is given for $b[7,8]$.

To determine the dynamic characteristics in terms of natural frequencies and mode shapes based on the linear time-invariant (LTI) assumption, a clutch stiffness value of $k_{C}=1838.0 \mathrm{Nm} \cdot \mathrm{rad}^{-1}$ is employed in Equation (5a) from Table 3, since the $4^{\text {th }}$ stage of torsional spring is operating under the given excitation condition. The natural frequencies based on the eigensolutions are $f_{1}=7.5 \mathrm{~Hz}$, $f_{2}=60.6 \mathrm{~Hz}$, and $f_{3}=273 \mathrm{~Hz}[7,8]$. To focus on impact phenomena such as gear rattle, the main resonance regime centers on $f_{2}=60.6 \mathrm{~Hz}$, because the physical driveline with a 4-cylinder engine is normally excited by gear impacts between $700 \mathrm{RPM}(23.3 \mathrm{~Hz})$ and $3000 \mathrm{RPM}(100 \mathrm{~Hz})[7,8]$. Thus, the frequency will be normalized with $f_{2}=60.6 \mathrm{~Hz}$ in further calculations. The driveline shown in Figure 1 is assumed to be in steady state given the sinusoidal excitations.

\section{HBM and Numerical Simulation (NS) with Jumping Phenomena}

By employing the HBM from prior studies [9,22], the nonlinear system responses with multiple locations of nonlinearities such as multi-stage clutch damper and gear backlash can be simulated based on the Galerkin scheme. Figure 3 shows a comparison of the HBM and numerical simulation (NS) results with focus on the maximum, mean, and minimum values in the time history of the relative displacement 
of the unloaded gear pair $\delta_{4}(\mathrm{t})$, which can be calculated with the following equation. In this simulation, the normalized frequency $\bar{\omega}\left(=\omega / \omega_{n}\right)$ is used:

$$
\delta_{4}(t)=R_{\text {iu }} \theta_{i}(t)+R_{\text {ou }} \theta_{\text {ou }}(\mathrm{t})
$$

To simulate the time responses using NS, the modified Runge-Kutta method has been employed [7,8]. Since the gear impact phenomenon consists of many super-harmonics, the simulation becomes more reliable as the number of harmonics $N_{\max }$ is increased [8]. However, this study is mainly limited to $N_{\max } \leq 6$ due to calculation time and convergence problems, which occur when the number of harmonics is increased beyond 6 , such as $N_{\max }=8$ or 12 . Here, $\eta$ indicates the sub-harmonic index.

The input torque $T_{E}(t)$ with $N_{\max }$ is calculated using the mean value of the input torque $T_{m}$ and the alternating part of the engine torque $T_{p i}$ as follows:

$$
T_{E}(t)=T_{m}+\sum_{i=1}^{N_{\max }} T_{p i} \cos \left(i \omega_{p} t+\phi_{p i}\right)
$$

In this equation, $\omega_{p}$ and $\phi_{P i}$ are the firing frequency and phase, respectively. Directly measured values from an engine dynamometer test of $T_{m}=168.9 \mathrm{Nm}, T_{p i}=251.5 \mathrm{Nm}$, and $\phi_{P i}=-1.93 \mathrm{rad}$ are employed for the HBM analysis [7,8].

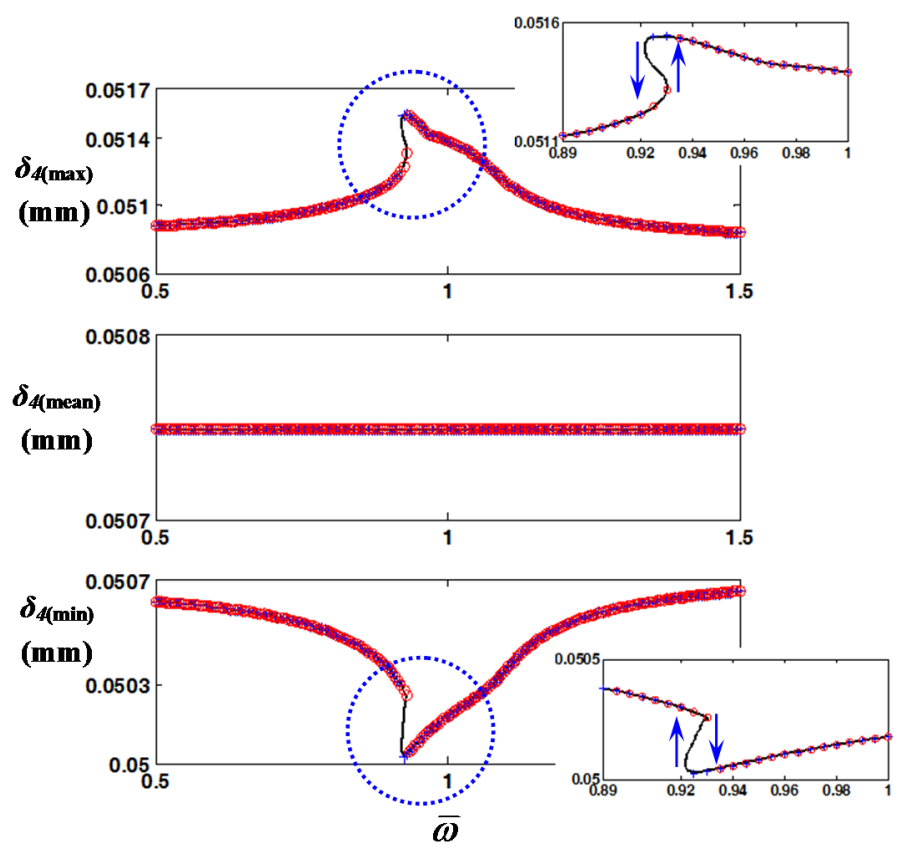

Figure 3. Comparison of the HBM $\left(N_{\max }=6\right.$ and $\left.\eta=2\right)$ results with the NS results in the frequency domain. Key: — HBM; 0 , NS by frequency up-sweeping;,+ NS by frequency down-sweeping.

To estimate the dynamic behaviors of the impact pair, the damping matrix is constructed using the assumed modal damping ratio $\zeta=0.02$, since it is much difficult to physically measure [7]. In addition, drag torques of Case IV in Table 4 are used for the baseline for this study since there is no impact with Case IV which will be explained more in detail. The drag torque values are given by assuming 4 different cases: Case I, vehicle normal driving condition; Case II, severe dynamic conditions of gearbox system; Case III, severe gear impact condition; Case IV, the most stable condition. 
Table 4. Employed properties for the drag torques for various conditions $[7,8]$.

\begin{tabular}{ccccc}
\hline \multirow{2}{*}{ Drag torque (Nm) } & \multicolumn{4}{c}{ Value } \\
\cline { 2 - 5 } & Case I & Case II & Case III & Case IV \\
\hline Drag torque on the input shaft, $T_{D i}$ & 75.0 & 11.9 & 48.4 & 30.8 \\
Drag torque on the output shaft, $T_{D o}$ & 57.9 & 9.2 & 37.4 & 23.8 \\
Drag torque on the unloaded gear, $T_{D u}$ & 3.9 & 3.1 & 2.5 & 7.2 \\
Drag torque on the vehicle, $T_{D V E 2}$ & 57.3 & 189.0 & 114.6 & 143.2 \\
\hline
\end{tabular}

As shown in Figure 3, the HBM and NS results in the frequency domain match well with each other. However, there is a large difference in the calculation times. For example, the HBM takes only 1 minute and 29 seconds, but the NS requires 3 hours. $N_{\max }=6$ and $\eta=2$ are employed for the HBM. Here, $\eta$ is limited to 2 by assuming that only period-doubling effect can be marginally observed in a practical system. Figure 4 compares the results in the time domain under frequency up- and down-sweeping conditions. By focusing on the normalized frequency $\bar{\omega}=0.93(=56.5 \mathrm{~Hz})$, where the jumping phenomenon is observed in Figure 3, the system responses differ considerably depending on the initial conditions and frequency sweeping directions. For example, as shown in Figure $4 \mathrm{~b}$, the dynamic behavior of $\delta_{4}(t)$ under the frequency down-sweeping condition includes more super-harmonic terms than $\delta_{4}(t)$ under the frequency up-sweeping condition given in Figure 4a. This is clearly shown at the peaks of the time history $\delta_{4}(t)$.

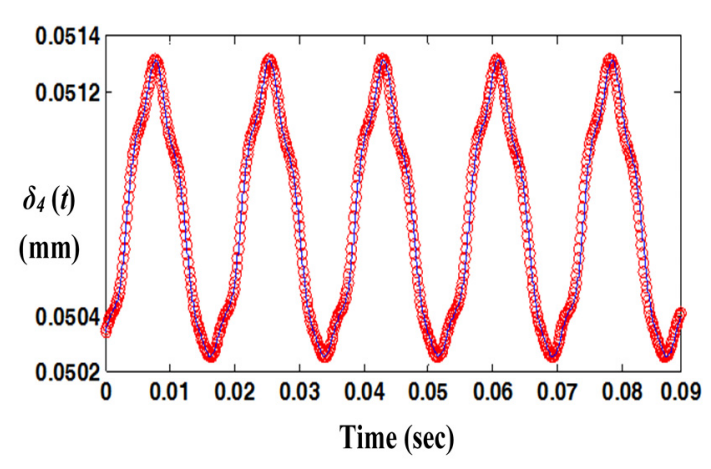

(a)

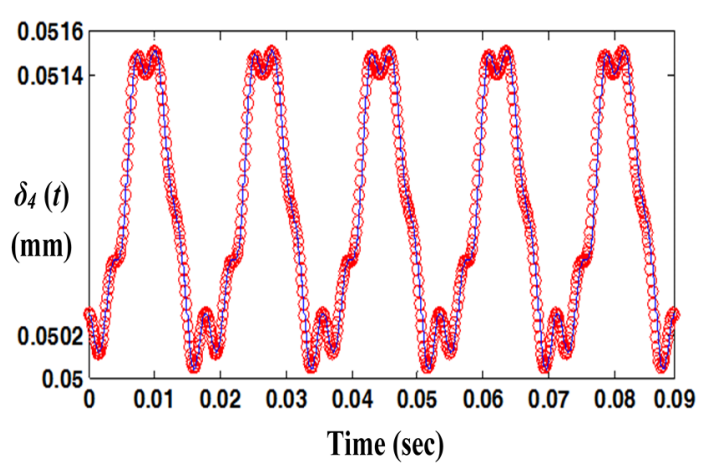

(b)

Figure 4. Comparison of the $\operatorname{HBM}\left(N_{\max }=6\right.$ and $\left.\eta=2\right)$ results with the NS results in the time domain at $56.5 \mathrm{~Hz}$ : (a) time histories of $\delta_{4}(t)$ under frequency up-sweeping; (b) time histories of $\delta_{4}(t)$ under frequency down-sweeping. Key: O, HBM; —- NS.

Figure 5 explains the variation in the max, mean, and min values of $\delta_{4}(t)$ from the HBM in the frequency domain when the number of harmonics is increased. To clearly observe the nonlinear behaviors of gear impact, a damping ratio of $1.3 \%$ is used, with which severe impact phenomena such as double-sided impact can be observed [7,8]. As shown in Figure 5, the maxima and minima at resonance and the shape of the backbone are significantly affected by $N_{\max }$. For example, the maximum and minimum values for $N_{\max }=1$ or $N_{\max }=3$ along with sub-harmonic index $\eta=1$ [9,22] are considerably smaller than those with $N_{\max }=6$ and $\eta=1$ or $N_{\max }=6$ and $\eta=2$.

When the jumping phenomena for the gear impact occur, the system response shows considerably different tracks depending on the frequency sweeping directions with $N_{\max }=6$ and $\eta=2$, as shown in 
Figure 5. When the system is under frequency up-sweeping, the value of the system response $\delta_{4(\max )}$ increases following the black dotted line until $\bar{\omega}\left(=\omega / \omega_{n}\right)$ reaches a value slightly larger than 0.92 .

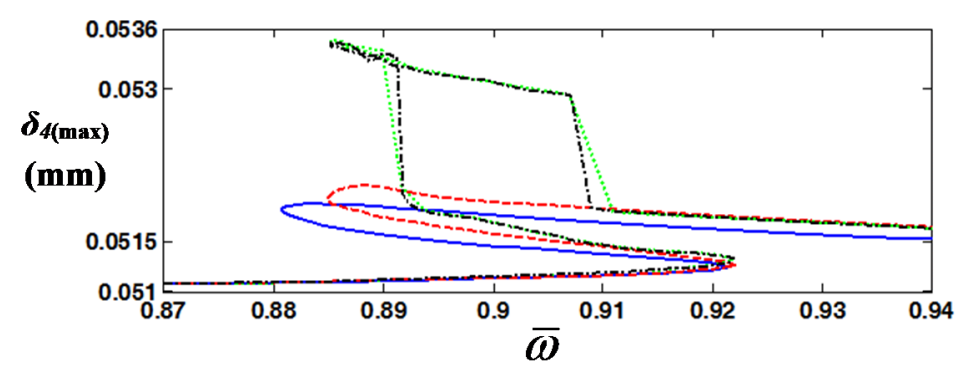

(a)

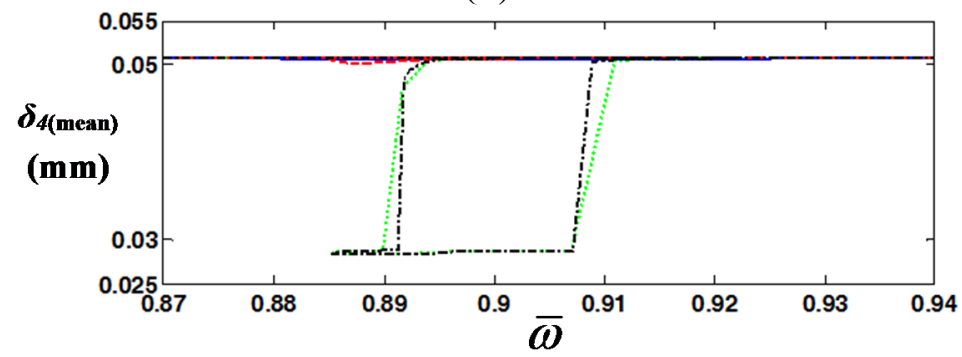

(b)

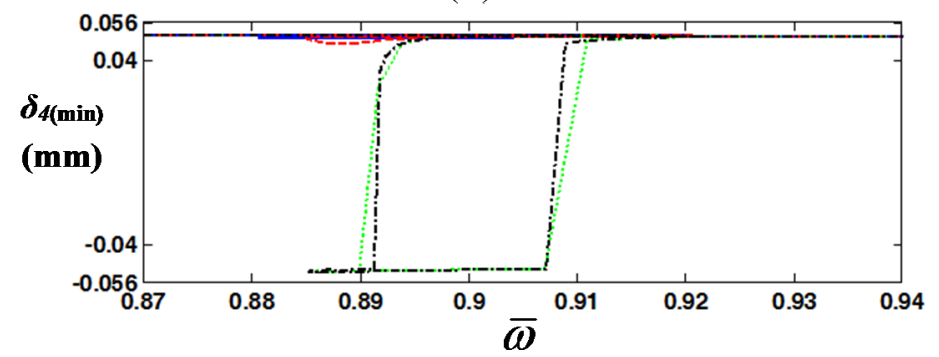

(c)

Figure 5. Max, mean, and min values of $\delta_{4}(t)$ from the HBM for 4 cases with different numbers of harmonics: (a) $\delta_{4}(t)_{(\max )}$; (b) $\delta_{4}(t)_{(\operatorname{mean})}$; (c) $\delta_{4}(t)_{(\min )}$. Key:,$- N_{\max }=1$ and $\eta=1 ;---, N_{\max }=3$ and $\eta=1 ; \cdots \ldots . . ., N_{\max }=6$ and $\eta=1 ;---, N_{\max }=6$ and $\eta=2$.

However, under frequency down-sweeping, the system responses have two jumping regimes, where jumping up occurs around $\bar{\omega}=0.91$ and jumping down occurs around $\bar{\omega}=0.885$. This dynamic characteristic reflects behavior similar to that observed in a prior study with a single gear pair [23]. Thus, the simulation results shown in Figure 5 prove that the gear impact analysis with an individual component can be reasonably extended to the simulation conditions in situ. In addition, the key factors to reduce the vibro-impacts can be determined easily based on the practical model with six DOF compared with a previously suggested component model [23]. In the next sections, the criteria with respect to the vibro-impacts will be investigated and discussed based on the simulation results.

\section{Impact Pair Analysis with Key Parameters}

Gear impacts in a geared system occur under various driving conditions corresponding to the drag torque properties listed in Table 4 [7]. The drag torques are estimated based on the results of a prior study $[7,8]$ by assuming that the gearbox is operated under different loading conditions, which have 
significant effects on the drag torque for the vehicle $T_{D V E 2}$. The other drag torques $T_{D i}, T_{D o}$, and $T_{D u}$ can be evaluated as suggested previously [7].

The simulated results for different drag torque conditions are compared in Figure 6. The results from Case I, II and III show the clear nonlinear impacts compared with Case IV where there is no impact. Case I is estimated by assuming that the vehicle system is operated under normal conditions. Case II involves the gearbox system being affected by double-sided impact. Case III shows the most severe gear impacts compared to the other cases since it shows double-sided impact as well as wider gear impact range. For example, even though Case II shows the same maxima of $\delta_{4(\max )}$ and minima of $\delta_{4(\min )}$ as Case III, the gear impact range for Case III is wider than for Case II.

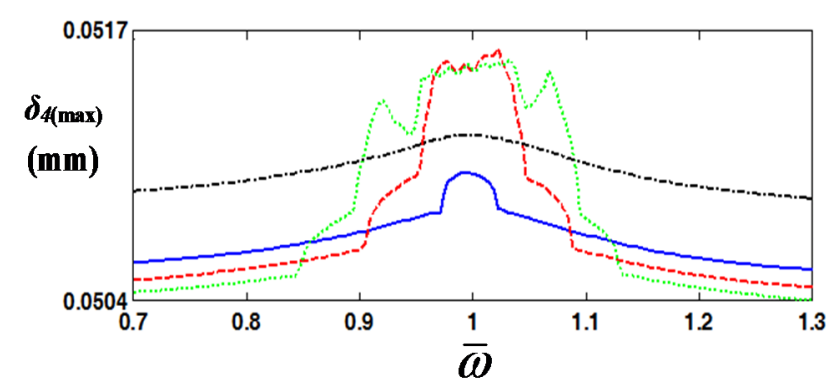

(a)

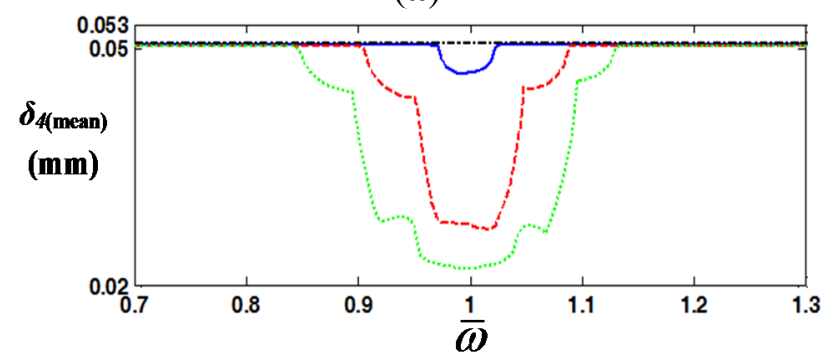

(b)

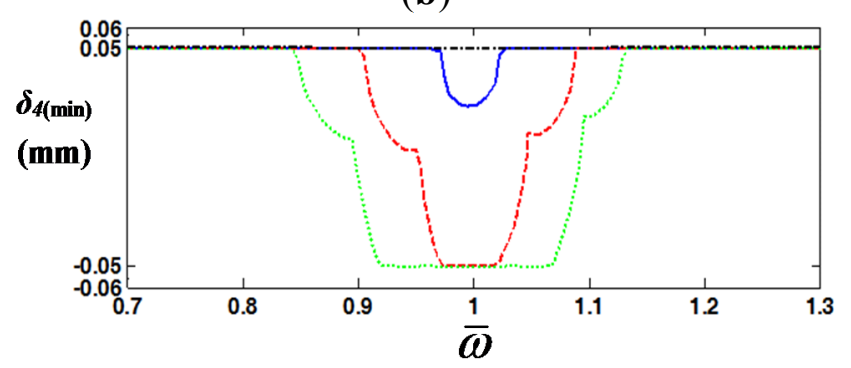

(c)

Figure 6. Max, mean, and min values of $\delta_{4}(t)$ from the HBM for various drag torque conditions: (a) $\delta_{4}(t)_{(\max )}$; (b) $\delta_{4}(t)_{(\operatorname{mean})}$; (c) $\delta_{4}(t)_{(\mathrm{min})}$. Key: — C Case I; ——- Case II; …....., Case III; - - - Case IV.

With focus on the gear impact, these vibro-impacts are significantly affected by the drag torque values on the unloaded gear $T_{D u}$, as shown in Table 4 . Thus, only Case IV overcomes the gear impacts, as shown in Figure 6. Based on the results, the gear impacts are related to the drag torque of the vehicle and the unloaded gear itself, which extends the gear rattle criteria concepts by including $T_{D V E 2}$. Thus, it will be clearly shown in the bifurcation diagram in the later section.

Figures 7-10 show the results of parametric studies with key property values such as the damping ratio $\zeta$, clutch stiffness $k_{C 4}$, and inertias of the flywheel $I_{f}$ and unloaded gear $I_{o u}$, given the drag torques 
of Case IV listed in Table 4. Figure 7 compares the HBM results for three modal damping ratios of $1.3 \%$, $1.5 \%$, and $2 \%$. As the damping value increases, the gear impacts are improved from double-sided impact to no-impact conditions [7-9]. In addition, the backbone curve can be anticipated with the resonance regimes easily. Figure 8 shows the effects of the clutch stiffness $k_{C 4}$ on the gear impacts given the modal damping ratio of $1.3 \%$. When $k_{C 4}$ is reduced, the gear impacts are improved from double-sided to single-sided impacts. However, changing the stiffness values affects the resonance regimes, because $k_{C 4}$ is significantly related to the natural frequencies of the torsional system $[7,8,22]$.

Figures 9 and 10 compare the gear impact conditions for the various inertias of the flywheel and unloaded gear. As clearly shown, increasing the inertia of the flywheel or decreasing the inertia values of the unloaded gear improves the gear impact conditions. Moreover, there are distinct differences in the HBM results in relation to these values. For example, the increase in $I_{f}$ changes the resonance area of

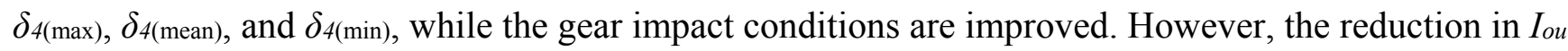

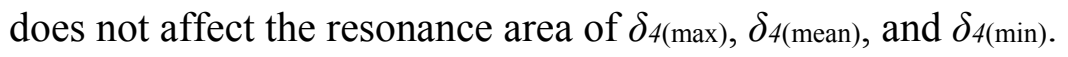
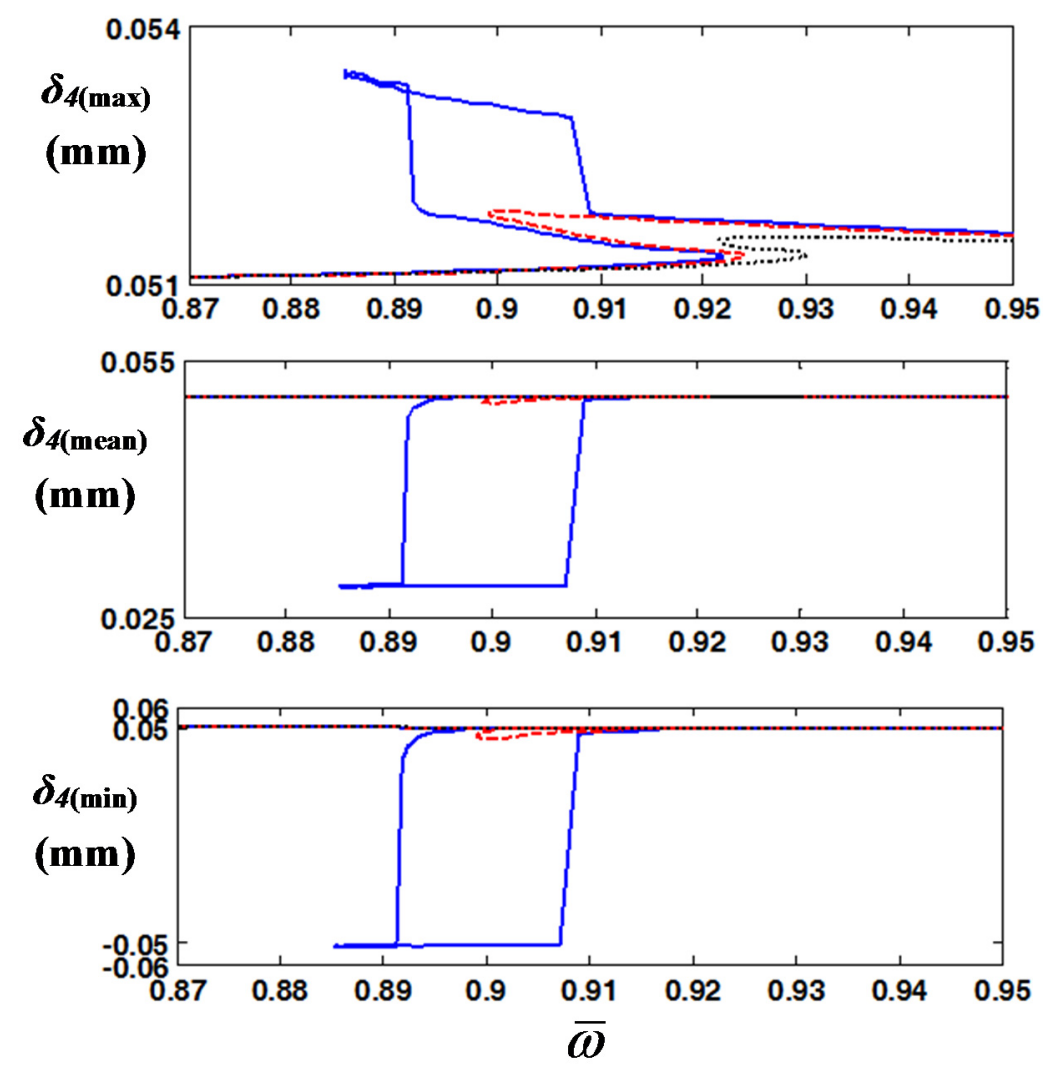

Figure 7. Max, mean, and min values of $\delta 4(t)$ from the $\operatorname{HBM}\left(N_{\max }=6\right.$ and $\left.\eta=2\right)$ for 3 values of damping ratio. Key:,$- \zeta=0.013 ;---, \zeta=0.015 ; \cdots \cdots \cdots \cdot \cdots, \zeta=0.02$. 

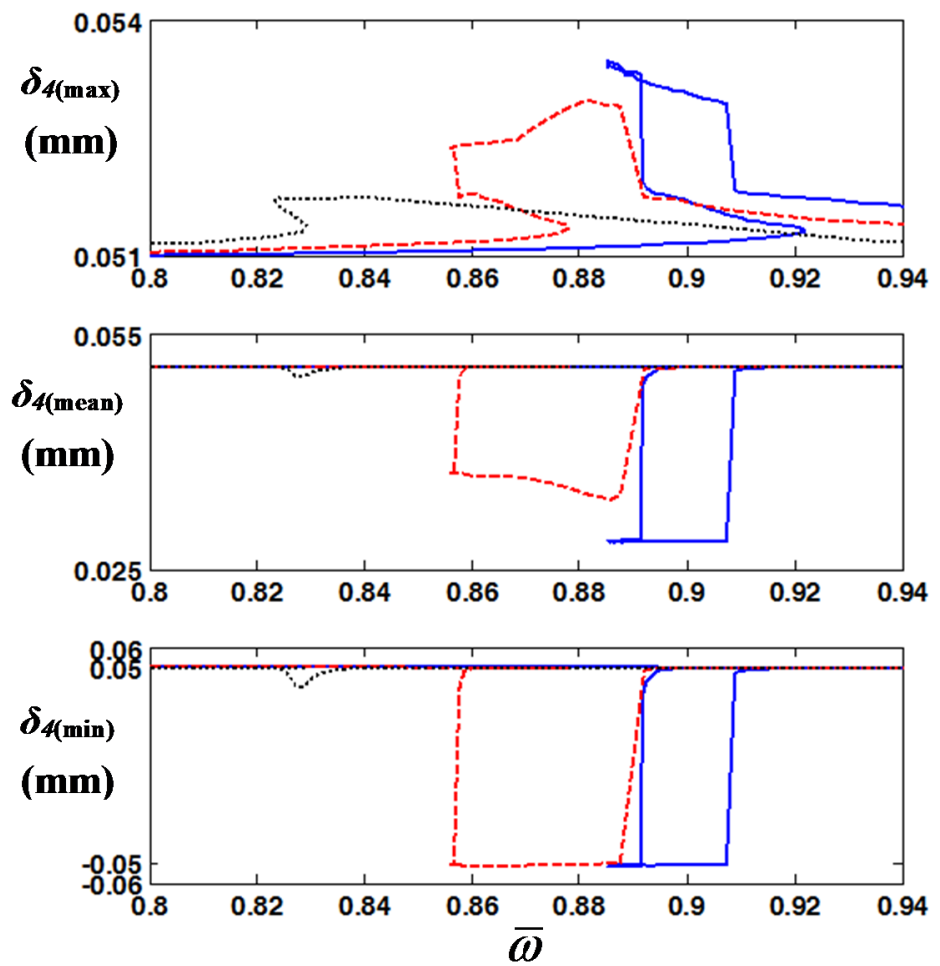

Figure 8. Max, mean, and min values of $\delta_{4}(t)$ from the HBM $\left(N_{\max }=6\right.$ and $\left.\eta=2\right)$ for 3 values of clutch stiffness. Key: —, $k_{C 4}=1838 \mathrm{Nm} / \mathrm{rad} ;-\longrightarrow, k_{C 4}=1562.3 \mathrm{Nm} / \mathrm{rad} ; \cdots . . . . . . .$. , $k_{C 4}=1279.2 \mathrm{Nm} / \mathrm{rad}$.
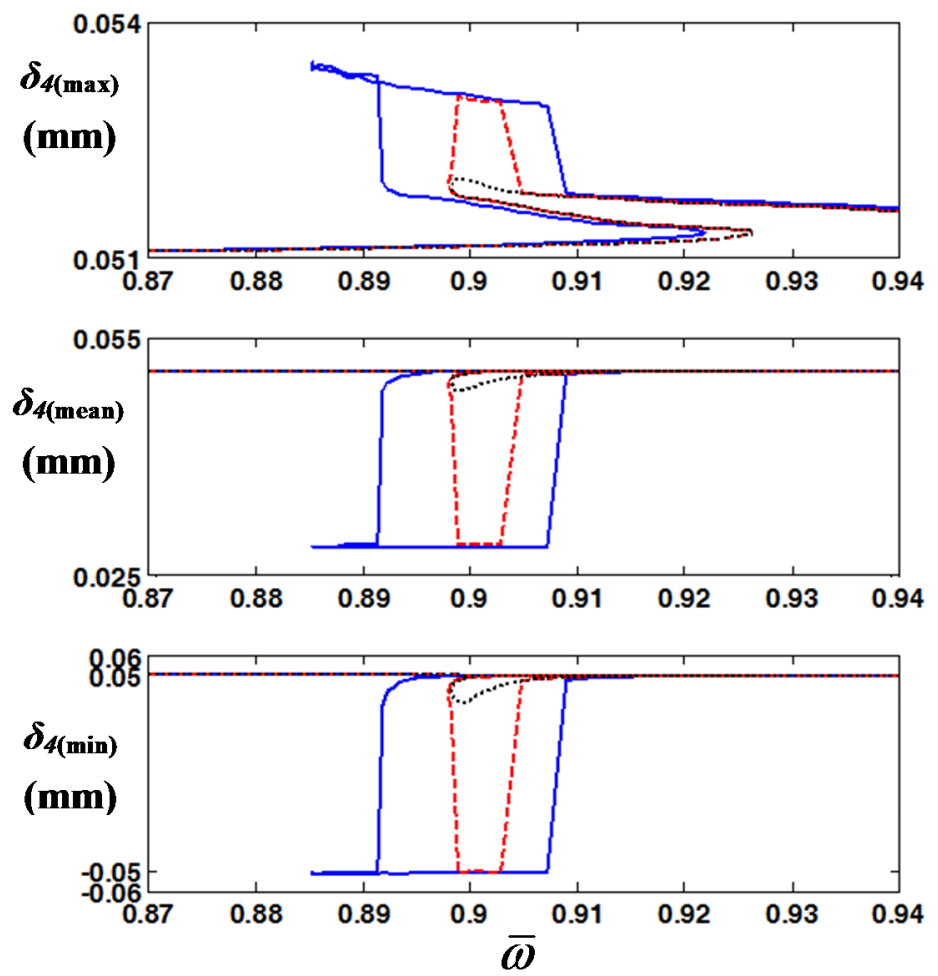

Figure 9. Max, mean, and min values of $\delta_{4}(t)$ from the $\operatorname{HBM}\left(N_{\max }=6\right.$ and $\left.\eta=2\right)$ for 3 values of inertia of flywheel $I_{f}$. Key: - $I_{f}=0.1376 \mathrm{~kg} \cdot \mathrm{m} ;---, I_{f}=0.1433 \mathrm{~kg} \cdot \mathrm{m} ; \cdots \cdots \cdots \cdot . .$. $I_{f}=0.1434 \mathrm{~kg} \cdot \mathrm{m}$. 

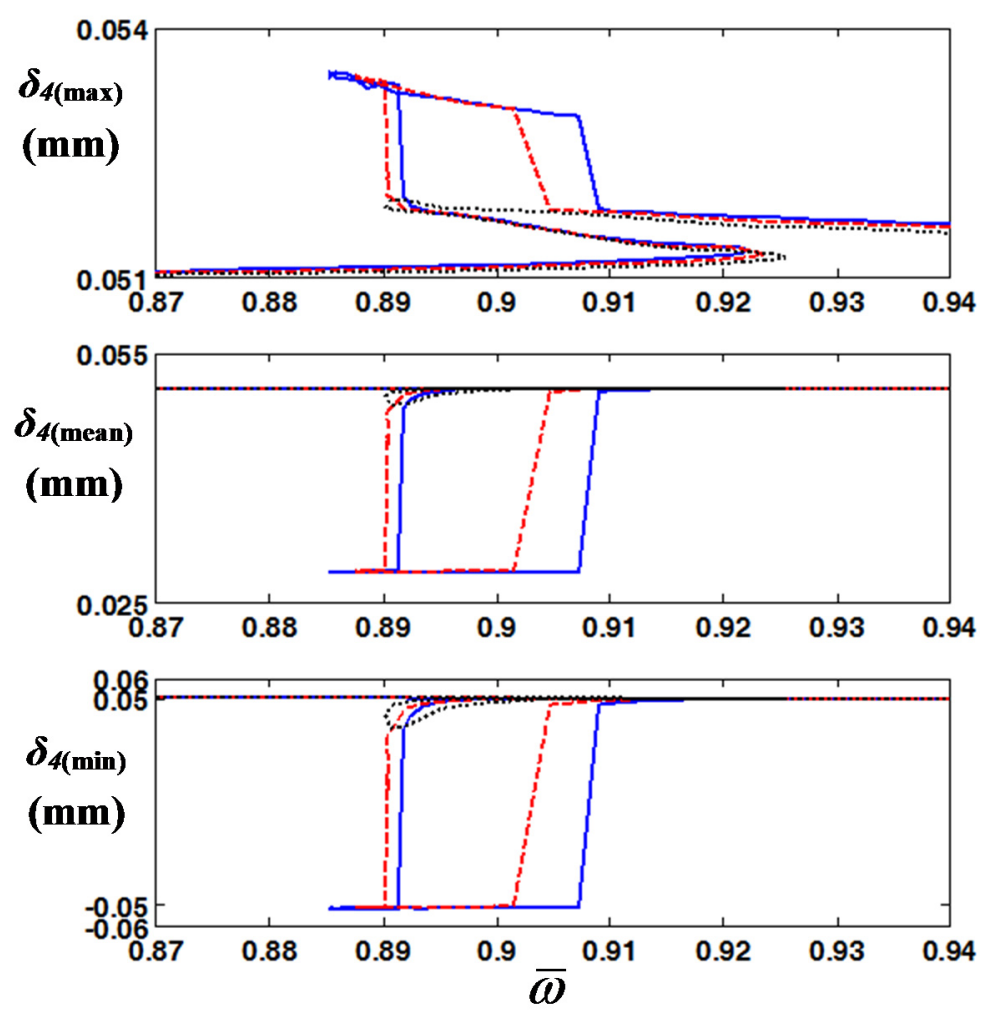

Figure 10. Max, mean, and min values of $\delta_{4}(t)$ from the $\operatorname{HBM}\left(N_{\max }=6\right.$ and $\left.\eta=2\right)$ for 3 values of inertia of unloaded gear $I_{o u}$. Key: —, $I_{o u}=5.23 \times 10^{-4} \mathrm{~kg} \cdot \mathrm{m} ;---, I_{o u}=4.97 \times 10^{-4} \mathrm{~kg} \cdot \mathrm{m}$; ………, $I_{o u}=4.61 \times 10^{-4} \mathrm{~kg} \cdot \mathrm{m}$.

Overall, based on the HBM results shown in Figures 7-10, the gear impact conditions still follow the previously suggested gear rattle criteria $[7,8]$. The vibro-impact conditions are improved with changes of the resonance regimes and key parameters such as $k_{C 4}$ and $I_{f}$ based on the HBM, which was not discussed in prior studies. Thus, these parametric studies can give design concepts to avoid gear impacts and system-level vibration problems.

\section{Investigation of Bifurcation Diagrams with Focus on Vibro-impacts}

Based on the case studies in Section 4, bifurcation diagrams can be drawn by fixing the specific excitation frequency to $\bar{\omega}=0.9$ where the double-sided impact occurs. The gear impact conditions can be mapped efficiently by comparing the bifurcation phenomena with gear rattle areas using the values

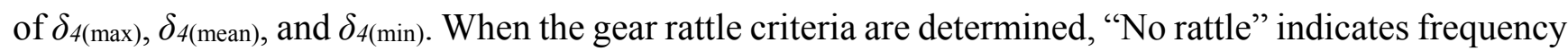
areas without any vibro-impacts at any initial conditions. "Rattle" indicates that at least one vibro-impact exists in any condition, either single- or double-sided impact. Figure 11 includes useful information such as the bifurcation and gear rattle regimes for given drag torques on the unloaded gear $T_{D u n}$, where the subscript $n$ indicates the given value of 7.2 Nm for Case IV, as listed in Table 4.

Here, the unstable values marked by a red cross in Figure 11 are estimated by employing Hill's method and the jumping phenomena occur at these areas as shown in Figure 2 [2]. Based on the simulated results, when $T_{D u}$ is increased, the gear impacts decrease and reach the no-impact condition in the region of $T_{D u} / T_{D u n}>1.35$. And this is correlated well with the prior studies [7,8]. However, the bifurcation still remains for the entire range of $T_{D u} / T_{D u n}$. This means that $\delta_{4}(t)$ shows considerably different dynamic behaviors 
depending on the values of $T_{D u} / T_{D u n}$ and the initial conditions. The red lines in Figure 11 indicate the marginal area of gear backlash with a value of $0.05 \mathrm{~mm}$ at $b / 2$ or $-0.05 \mathrm{~mm}$ at $-b / 2$. Figures $12-14$ show the bifurcation diagrams for the key parameters considered in Section 4.

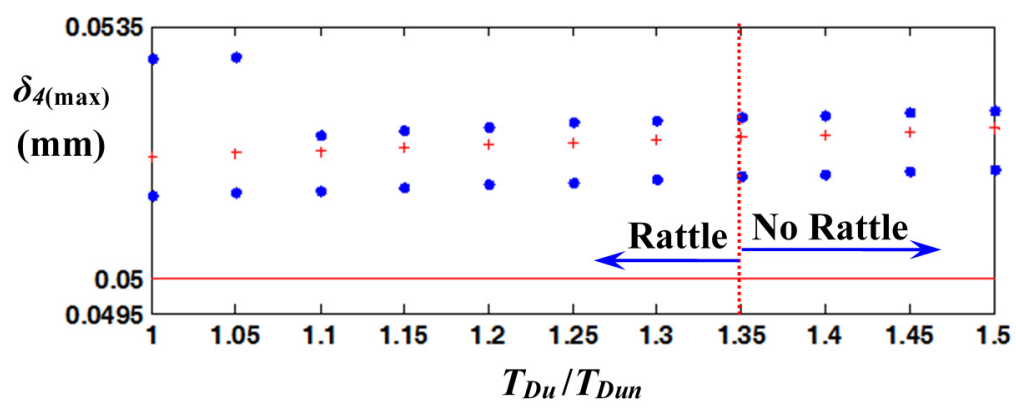

(a)

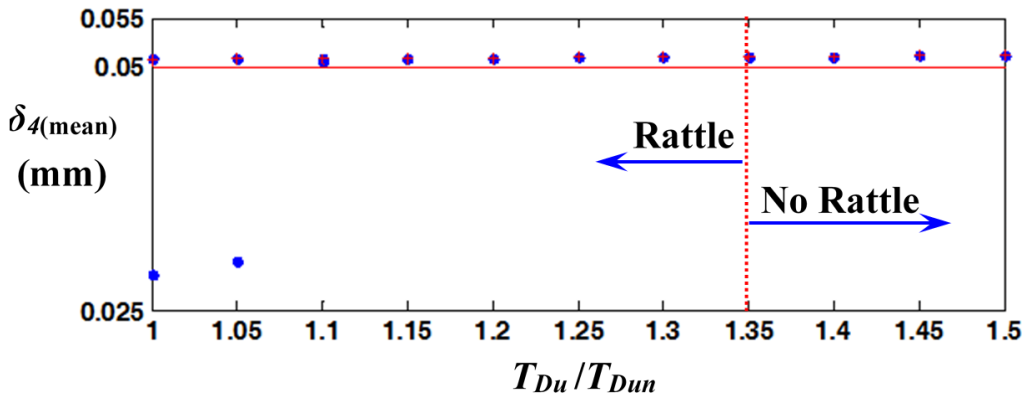

(b)

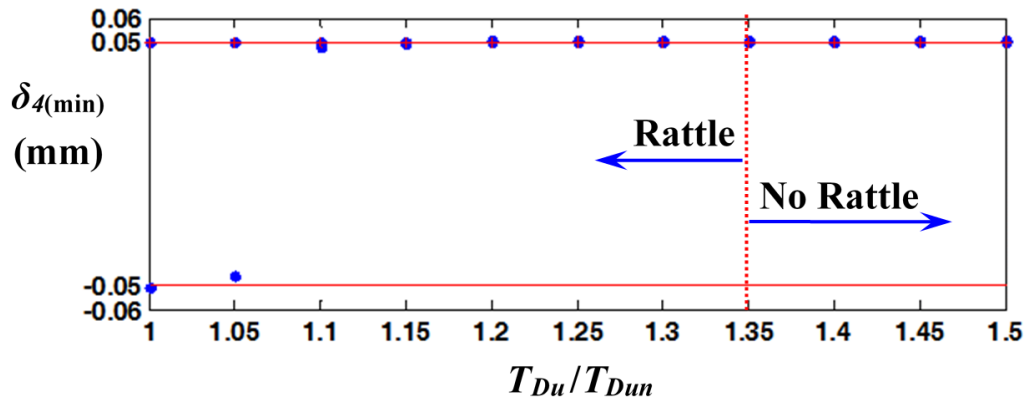

(c)

Figure 11. Bifurcation diagrams of max, mean, and min values of $\delta_{4}(t)$ for drag torque on the unloaded gear: (a) $\delta_{4}(t)_{(\max )}$; (b) $\delta_{4}(t)_{(\operatorname{mean})}$; (c) $\delta_{4}(t)_{(\min )}$. Key: $\bullet$, stable value; $\boldsymbol{+}$, unstable value; - , marginal area of gear backlash.

In general, the gear impact phenomena are closely related to the bifurcation regimes, along with the initial or frequency sweeping conditions. Figure 12 shows the bifurcation results for damping ratios. The employed modal damping $\zeta_{n}$ is 0.013 , and the bifurcation disappears when $\zeta / \zeta_{n}$ is greater than 1.18, where the gear impact is also resolved.

Figure 13 shows bifurcation diagrams for $k_{C 4} / k_{C 4 n}$, where $k_{C 4 n}$ is $1838.0 \mathrm{Nm} / \mathrm{rad}$. The bifurcation disappears when $k_{C 4} / k_{C 4 n}$ is lower than 0.9 , and the vibratory problems due to gear impacts are resolved when $k_{C 4} / k_{C 4 n}$ is less than 0.8 . Figure 14 shows bifurcation diagrams for $I_{f} / I_{f n}$, where $I_{f n}=1.38 \times 10^{-1} \mathrm{~kg} \cdot \mathrm{m}^{2}$. The bifurcation and gear impacts do not occur when $I_{f} / I_{f n}>1.05$. In contrast, Figure 15 shows bifurcation diagrams for $I_{o u} / I_{o u n}$ with the reverse tendency compared to the bifurcation for $I_{f} / I_{f n}$. For example, the bifurcation and gear impacts disappear when $I_{o u} / I_{o u n}<0.66$. 

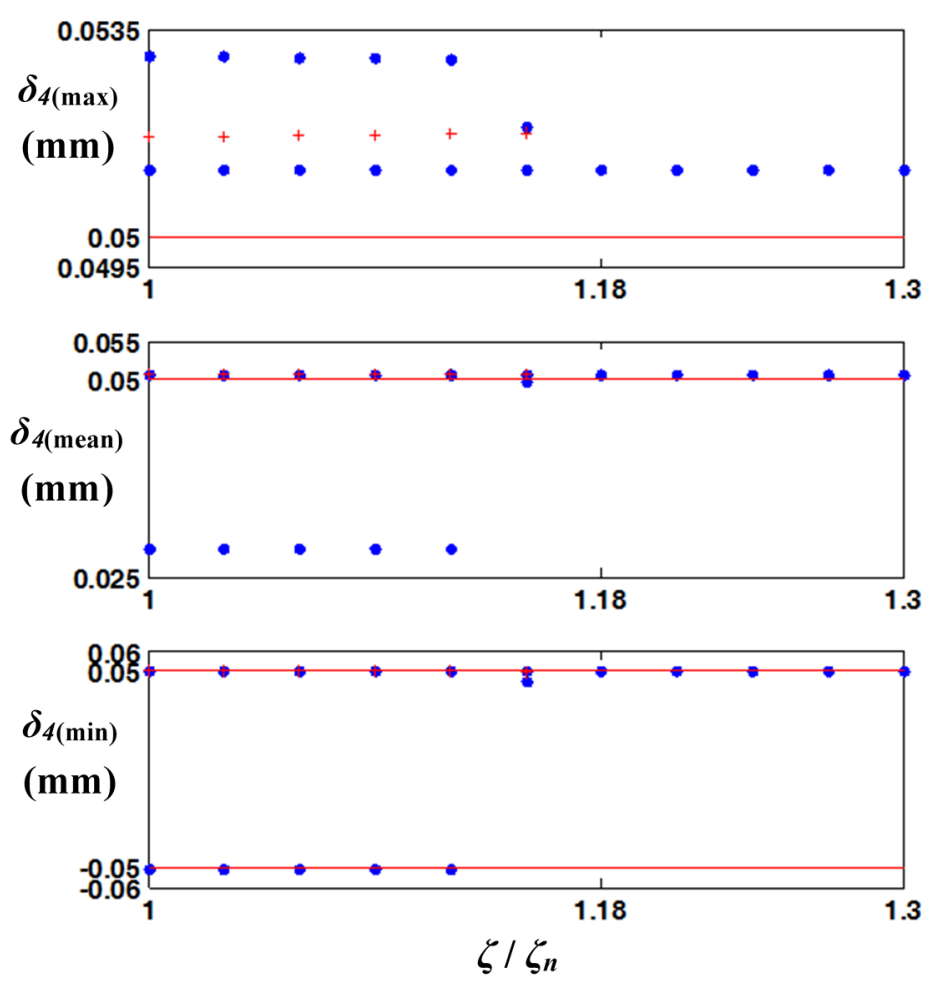

Figure 12. Bifurcation diagrams of max, mean, and min values of $\delta_{4}(t)$ for damping ratio. Key: •, stable value; +, unstable value; ——, marginal area of gear backlash.
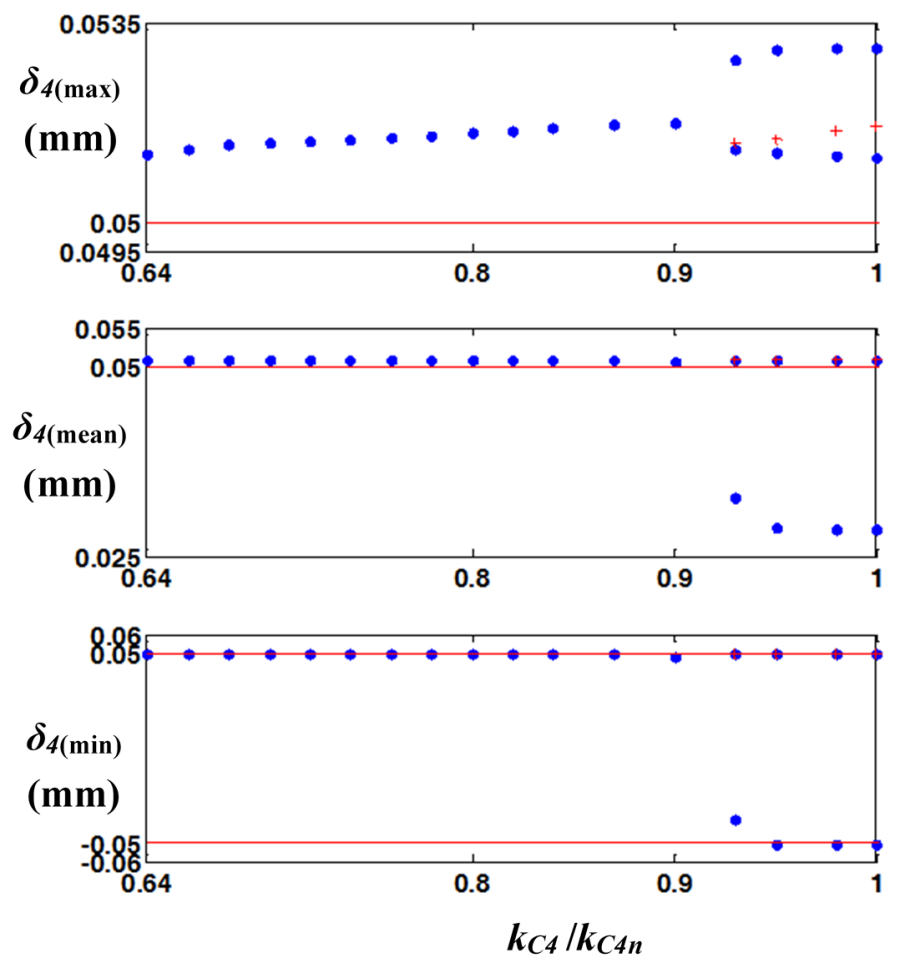

Figure 13. Bifurcation diagrams of max, mean, and min values of $\delta_{4}(t)$ for clutch stiffness. Key: $\bullet$, stable value; + , unstable value; __, marginal area of gear backlash. 

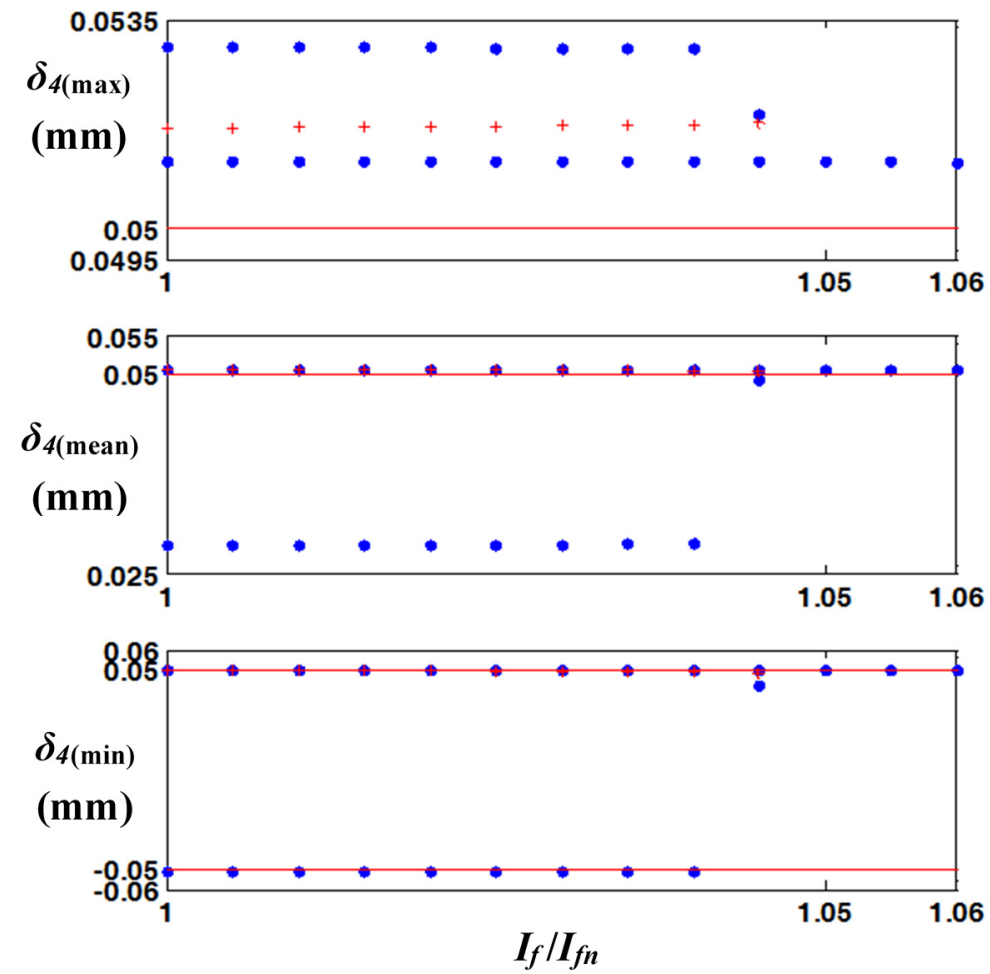

Figure 14. Bifurcation diagrams of max, mean, and min values of $\delta_{4}(t)$ for inertia of flywheel. Key: $\bullet$, stable value; +, unstable value; —_, marginal area of gear backlash.
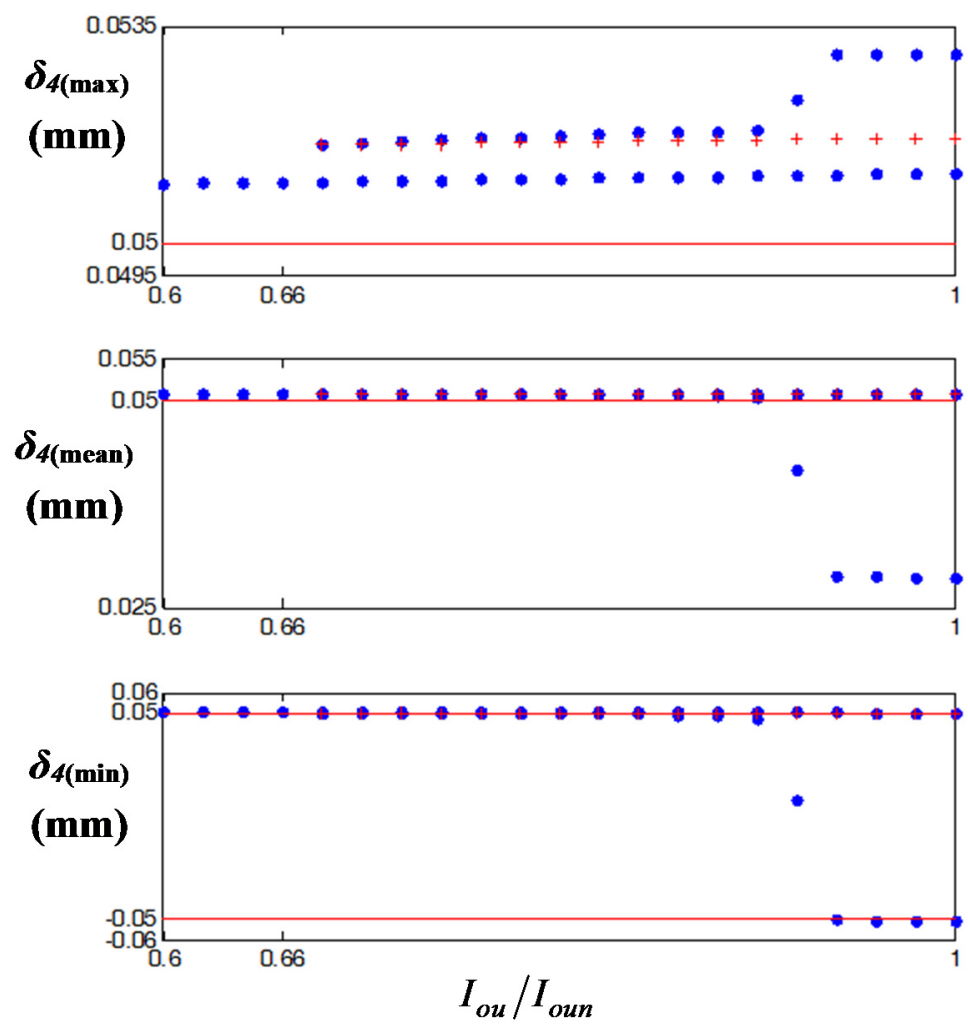

Figure 15. Bifurcation diagrams of max, mean, and min values of $\delta_{4}(t)$ for the inertia of the unloaded gear. Key: $\bullet$, stable value; + , unstable value; —_, marginal area of gear backlash. 
Overall, based on the examination of the bifurcation diagrams for the key parameters, the gear impacts can be resolved by adapting relevant parameters, as suggested in prior studies [7-9]. Thus, the vibration problems caused by the gear impacts can be resolved by increasing $T_{D U}, I_{f}$, and $\zeta$ or decreasing $k_{C 4}$ and Iou. Moreover, the bifurcation sometimes does not disappear where the gear impacts are resolved, as shown in Figure 11, where the bifurcation can be observed for the entire range of $T_{D u} / T_{D u n}$, including the non-impact region. On the other hand, the bifurcation is directly correlated with the impact phenomena in some cases. For example, as shown in Figure 15, the bifurcation and gear impact occur (or disappear) at the same $I_{\text {ou/ }}$ Ioun $_{\text {o }}$

\section{Conclusions}

This article investigated the gear impact phenomena in both the frequency and time domains with a 6-DOF nonlinear torsional system model using the HBM. In addition, the dynamic characteristics of the gear impacts have been examined and explained using bifurcation diagrams based on parametric studies with respect to key parameters such as the clutch stiffness, drag torque, and inertias of the flywheel and unloaded gear. The specific contributions of this research are summarized as follows.

First, the dynamic behaviors of impact pairs in a physical system have been investigated by implementing piecewise-type nonlinearities at multiple points. This study advanced a prior study [23] by extending the study scope from focusing on impact pairs only to the vibro-impacts in a gearbox system such as vehicle driveline or wind turbines under sinusoidal excitation. Second, the effects of key parameters on the vibro-impacts have been examined using the HBM. This study provides understanding of the relationships between the impact phenomena of gear pairs and their relevant parameters, and they can be applied to improve the vibratory conditions. Also, this concept can be employed to the design issues when the wind turbine system is constructed since this macro-system always contains the durability problems under the severe input conditions related to the vibration problems. Third, the impact phenomena have been mapped using bifurcation diagrams focused on key parameters. Thus, the vibro-impact has been clearly assessed, and the effects of the key parameters have been examined with bifurcation diagrams. Using bifurcation diagram also will lead to increasing the efficiency of designing the wind turbine system by assessing the properties of key parameters. However, this method needs to be correlated with experiments based on time-domain data, which is a subject for future study, including the development of adapted simulation method for the wind turbine.

\section{Acknowledgments}

This work was supported by the 2015 Yeungnam University Research Grant (215A580008).

\section{Author Contributions}

Jong-yun Yoon and Byeongil Kim initiated and developed the ideas related to this research work. Jong-yun Yoon and Byeongil Kim developed novel methods, derived relevant formulations, and carried out performance analyses and numerical analyses. Jong-yun Yoon wrote the paper draft under Byeongil Kim's guidance and Byeongil Kim finalized the paper. 


\section{Conflicts of Interest}

The authors declare no conflict of interest.

\section{References}

1. Padmanabhan, C.; Singh R. Spectral coupling issues in a two-degree-of-freedom system with clearance non-linearities. J. Sound Vib. 1992, 155, 209-230.

2. Kim, T.C.; Rook, T.E.; Singh R. Super- and sub-harmonic response calculation for a torsional system with clearance nonlinearity using the harmonic balance method. J. Sound Vib. 2005, 281, 965-993.

3. Kim, T.C.; Rook, T.E.; Singh R. Effect of nonlinear impact damping on the frequency response of a torsional system with clearance. J. Sound Vib. 2005, 281, 995-1021.

4. Kim, T.C.; Rook, T.E.; Singh R. Effect of smoothening functions on the frequency response of an oscillator with clearance non-linearity. J. Sound Vib. 2003, 263, 665-678.

5. Rook, T.E.; Singh R. Dynamic analysis of a reverse-idler gear pair with concurrent clearance. J. Sound Vib. 1995, 182, 303-322.

6. Comparin, R.J.; Singh R. Frequency response characteristics of a multi-degree-of-freedom system with clearance. J. Sound Vib. 1990, 142, 101-124.

7. Yoon, J.Y.; Singh R. Effect of multi-staged clutch damper characteristics on transmission gear rattle under two engine conditions. J. Automob. Eng. 2013, 227, 1273-1294.

8. Yoon, J.Y.; Lee, I.J. Nonlinear analysis of vibro-impacts for unloaded gear pairs with various excitation and system parameters. ASME J. Vib. Acous. 2014,136, 031010.

9. Yoon, J.Y.; Yoon, H.S. Nonlinear frequency response analysis of a multi-stage clutch damper with multiple nonlinearities. ASME J. Comput. Nonlinear Dyn. 2014, 9, 031007.

10. Peng, Z.K.; Lang, Z.Q.; Billings, S.A.; Tomlinson, G.R. Comparison between harmonic balance and nonlinear output frequency response function in nonlinear system analysis. J. Sound Vib. 2008, 311, 56-73.

11. Chen, Y.M.; Liu, J.K.; Meng, G. Incremental harmonic balance method for nonlinear flutter of an airfoil with uncertain-but-bounded parameters. Appl. Math. Model. 2012, 36, 657-667.

12. Al-shyyab, A.; Kahraman, A. Non-linear dynamic analysis of a multi-mesh gear train using multi-term harmonic balance method: sub-harmonic motions. J. Sound Vib. 2005, 279, 417-451.

13. Genesio, R.; Tesi, A. Harmonic balance methods for the analysis of chaotic dynamics in nonlinear systems. Automatica 1992, 28, 531-548.

14. Masiani, R.; Capecchi, D.; Vestroni, F. Resonant and coupled response of hysteretic two-degree-of-freedom systems using harmonic balance method. Int. J. Non-Linear Mech. 2002, 37, 1421-1434.

15. Raghothama, A.; Narayanan, S. Bifurcation and chaos in geared rotor bearing system by incremental harmonic balance method. J. Sound Vib. 1999, 226, 469-492.

16. Raghothama, A.; Narayanan, S. Bifurcation and chaos of an articulated loading platform with piecewise non-linear stiffness using the incremental harmonic balance method. Ocean Eng. 2000, 27, 1087-1107. 
17. Shen, Y.; Yang, S.; Liu, X. Nonlinear dynamics of a spur gear pair with time-varying stiffness and backlash based on incremental harmonic balance method. Int. J. Mech. Sci. 2006, 48, 1256-1263.

18. Wong, C.W.; Zhang, W.S.; Lau, S.L. Periodic forced vibration of unsymmetrical piecewise-linear systems by incremental harmonic balance method. J. Sound Vib. 2006, 48, 1256-1263.

19. Sundararajan, P.; Noah, S.T. Dynamics of forced nonlinear systems using shooting/arc-length continuation method-application to rotor systems. ASME J. Vib. Acous. 1997, 119, 9-20.

20. Sundararajan, P.; Noah, S.T. An algorithm for response and stability of large order non-linear systems-application to rotor systems. J. Sound Vib. 1998, 214, 695-723.

21. Royston, T.J.; Singh, R. Periodic response of mechanical systems with local non-linearities using an enhanced Galerkin technique. J. Sound Vib. 1996, 194, 243-263.

22. Yoon, J.Y.; Lee, H.I. Dynamic vibratory motion analysis of a multi-degree-of-freedom torsional system with strongly stiff nonlinearities. J. Mech. Eng. Sci. 2015, 229, 1399-1414.

23. Comparin, R.J.; Singh, R. Non-linear frequency response characteristics of an impact pair. J. Sound Vib. 1989, 134, 259-290.

(C) 2015 by the authors; licensee MDPI, Basel, Switzerland. This article is an open access article distributed under the terms and conditions of the Creative Commons Attribution license (http://creativecommons.org/licenses/by/4.0/). 